\title{
BIODISPONIBILIDADE DE FORMAS DE FÓSFORO ACUMULADAS EM SOLO SOB SISTEMA PLANTIO DIRETO ${ }^{(1)}$
}

\author{
Luciano Colpo Gatiboni $^{(2)}$, João Kaminski ${ }^{(3)}$, Danilo dos Santos \\ Rheinheimer $^{(4)}$ \& João Paulo Cassol Flores ${ }^{(5)}$
}

\begin{abstract}
RESUMO
A adição de fertilizantes ao solo provoca aumento dos teores de diversas formas de $\mathbf{P}$ com diferentes energias de ligação com os colóides. Isso tem sido observado na camada superficial de solos cultivados sob sistema plantio direto, porém há poucas informações sobre a disponibilidade de $\mathrm{P}$ dessas formas para as plantas. $\mathrm{O}$ objetivo do presente trabalho foi avaliar a biodisponibilidade das formas de $\mathbf{P}$ acumuladas em um solo cultivado sob sistema plantio direto com diferentes quantidades de $\mathbf{P}$ adicionado. Foram coletadas amostras da camada de $\mathbf{0}-\mathbf{0 , 1 0} \mathrm{m}$ de um Latossolo Vermelho distroférrico típico que recebeu 0, 180, 360, 540 e $720 \mathrm{~kg} \mathrm{ha}^{-1}$ de $\mathrm{P}_{2} \mathrm{O}_{5}$ em seis anos de cultivo. As amostras foram secas, moídas, acondicionadas em vasos com capacidade de $2 \mathrm{~kg}$ e submetidas a 15 cultivos sucessivos em casa de vegetação, sem reposição do $P$ absorvido pelas plantas. Antes dos cultivos e após cada três cultivos sucessivos, foram coletadas amostras de solo para análise. Foram realizadas análises de fracionamento das formas de $\mathbf{P}$ pelo método de Hedley. Os resultados mostraram que o $\mathrm{P}$ inorgânico extraído por $\mathrm{NaHCO}_{3} \quad 0,5 \mathrm{~mol} \mathrm{~L}^{-1}$ apresenta capacidade e velocidade de tamponamento semelhantes às do $\mathrm{P}$ disponível por resina trocadora de ânions, podendo ser considerado também como $\mathbf{P}$ lábil. Em longo prazo, todas as formas de $\mathbf{P}$ do solo atuam na sustentação do $\mathbf{P}$ absorvido pelas plantas; em solos com acúmulo de $\mathbf{P}$ pela adubação, as formas inorgânicas e orgânicas de $P$ atuam de forma semelhante no tamponamento do $\mathrm{P}$ absorvido pelas plantas. Em solos com baixa ou nenhuma adição de fertilizantes fosfatados, as formas orgânicas de $P$ são as principais mantenedoras do $\mathbf{P}$ absorvido pelas plantas.
\end{abstract}

Termos de indexação: disponibilidade, fracionamento, cultivos sucessivos, fósforo orgânico.

\footnotetext{
(1) Parte da Tese de Doutorado do primeiro autor. Trabalho financiado pelo CNPq-Pronex e FAPERGS. Recebido para publicação em junho de 2005 e aprovado em março de 2007.

(2) Professor adjunto do Departamento de Solos da Universidade do Estado de Santa Catarina - UDESC. Av. Luiz de Camões 2090 , CEP 88520-000 Lages (SC). Bolsista de Produtividade de Pesquisa do CNPq. E-mail: gatiboni@udesc.br

(3) Professor do Departamento de Solos da UFSM. E-mail: jk@smail.ufsm.br

(4) Professor do Departamento de Solos da UFSM. E-mail: danilor@smail.ufsm.br

${ }^{(5)}$ Doutorando do curso de Pós-graduação em Ciência do Solo da UFRGS. E-mail: joaopcflores@pop.com.br
} 


\title{
SUMMARY: BIOAVAILABILITY OF SOIL PHOSPHORUS FORMS IN NO- TILLAGE SYSTEM
}

\begin{abstract}
The addition of fertilizers to the soil increases $P$ contents in many forms of different bond energies with soil colloids. This has been observed in the soil surface layer under no tillage system, however there is little information about the availability of these $P$ forms to plants. The study aimed to evaluate the bioavailability of $P$ forms of a soil under no tillage with $P$ applications. Samples were collected $(0-10 \mathrm{~cm})$ in a Rhodic Hapludox that had been fertilized with $0,180,360,540$, and $720 \mathrm{~kg} \mathrm{ha}^{-1}$ of $\mathrm{P}_{2} \mathrm{O}_{5}$ over six years of cultivation prior to this study. The samples were dried, ground, filled in $2 \mathrm{~kg}$ pots and subjected to 15 successive crops without replacement of absorbed P. After every three successive crops, soil samples were collected for analysis. Phosphorus fractionation was analyzed by Hedley's method. Results showed that inorganic P extracted by $\mathrm{NaHCO}_{3} 0.5 \mathrm{~mol} \mathrm{~L}^{-1}$ has a buffering capacity similar to $P$ available by anion exchange resin, and can be considered labile $P$ as well. In the long term, all soil $P$ forms act in the soil solution buffer. In fertilized soils, the inorganic and organic $P$ forms act in a similar way buffering the absorbed $P$ by plants. In soils with little or no fertilization, the organic forms are the main $P$ source of plants.
\end{abstract}

Index terms: lability, fractionation, successive crops, organic phosphorus.

\section{INTRODUÇÃO}

O P do solo é distribuído em formas que variam com a natureza química do ligante e a energia de ligação entre o solo e este elemento. Quanto à natureza do ligante, o $\mathrm{P}$ pode ser encontrado como $\mathrm{P}$ orgânico diéster, $\mathrm{P}$ orgânico monoéster, $\mathrm{P}$ inorgânico em ligações com $\mathrm{Fe}, \mathrm{Al}, \mathrm{Ca}$, argilas silicatadas e óxidos, entre outras. Quanto à energia de ligação, o $\mathrm{P}$ pode estar associado a outros íons ou moléculas em ligações monodentadas, bidentadas e bionucleadas (Dalal, 1977; Parfitt, 1978). Assim, as formas de P do solo têm diferentes capacidades de dessorção e abastecimento da solução do solo, segundo sua natureza química e energia de ligação. Do ponto de vista da Fertilidade do Solo, independentemente da natureza química, o $\mathrm{P}$ é dividido de acordo com a facilidade de reposição da solução do solo.

Para estudos do acúmulo e da dessorção de formas de $\mathrm{P}$ segundo sua labilidade, diversos autores têm utilizado a técnica de fracionamento de $\mathrm{P}$ proposta por Hedley et al. (1982), que usa, seqüencialmente, extratores de menor à maior força de extração, os quais removem $\mathrm{P}$ inorgânico $(\mathrm{Pi})$ e orgânico $(\mathrm{Po})$ das formas mais disponíveis às mais estáveis. Com as modificações propostas por Condron et al. (1985), os extratores utilizados no fracionamento são, seqüencialmente, resina trocadora de ânions $\left(\mathrm{Pi}_{\mathrm{RTA}}\right)$, $\mathrm{NaHCO}_{3}$ 0,5 mol L-1 a pH 8,5 ( $\mathrm{Pi}_{\text {bic }}$ e $\left.\mathrm{Po}_{\text {bic }}\right)$, NaOH $0,1 \mathrm{~mol} \mathrm{~L}^{-1}\left(\mathrm{Pi}_{\text {hid }}\right.$ e $\left.\mathrm{Po}_{\text {hid }}\right), \mathrm{HCl} 1,0 \mathrm{~mol} \mathrm{~L}-1$ (Pi $\left.\mathrm{HCl}_{\mathrm{HCl}}\right)$, $\mathrm{NaOH} 0,5 \mathrm{~mol} \mathrm{~L}^{-1}\left(\mathrm{Pi}_{\text {hid }, 5}\right.$ e $\left.\mathrm{Po}_{\text {hido,5 }}\right)$ e digestão com $\mathrm{H}_{2} \mathrm{SO}_{4}+\mathrm{H}_{2} \mathrm{O}_{2}+\mathrm{MgCl}_{2}\left(\mathrm{P}_{\text {residual }}\right)$. As frações orgânicas (Po) são determinadas pela diferença entre o $\mathrm{P}$ total $\mathrm{e}$ o Pi em cada extrator.
Estudos que utilizam o fracionamento de Hedley têm mostrado que as frações orgânicas e inorgânicas de $\mathrm{P}$ no solo podem atuar como fonte ou dreno para a solução do solo, dependendo das suas características mineralógicas, das condições ambientais e da fertilização e do manejo do solo. Tiessen et al. (1984) e Beck \& Sanches (1994) observaram que em ecossistemas naturais a disponibilidade de $\mathrm{P}$ está intimamente relacionada à ciclagem das formas orgânicas, enquanto em solos pouco intemperizados ou fertilizados grande parte do $\mathrm{P}$ disponível é tamponada pelas frações inorgânicas lábeis. Por outro lado, em solos altamente intemperizados ou sem fertilização, esses autores observaram que a disponibilidade de $\mathrm{P}$ é altamente dependente das formas inorgânicas e orgânicas de labilidade intermediária. Guo \& Yost (1998) realizaram experimento em casa de vegetação utilizando solos com moderado e alto grau de intemperismo, nos quais realizaram 14 cultivos sucessivos de milho ou soja, com prévia adição de P. Os autores observaram que nos solos pouco intemperizados a depleção das formas lábeis de $\mathrm{Pi}\left(\mathrm{Pi}_{\mathrm{RTA}}\right.$ e $\left.\mathrm{Pi}_{\text {bic }}\right)$ causada pela absorção por plantas foi tamponada pelas frações menos lábeis $\left(\mathrm{Pi}_{\mathrm{HCl}}\right.$ e $\left.\mathrm{P}_{\text {residual }}\right)$. Por outro lado, nos solos altamente intemperizados o tamponamento foi feito pelas frações $\mathrm{Po}_{\text {bic }}$ e $\mathrm{Pi}_{\text {hid }}$. Esses autores relatam que, em médio e longo prazos, todas as formas de $\mathrm{P}$ do solo são disponíveis às plantas, mas não garantem que as taxas de absorção sejam suficientes para manter altos rendimentos.

Com a adição de fertilizantes fosfatados, há acúmulo de $\mathrm{P}$ em formas inorgânicas e orgânicas com diferentes graus de energia de ligação, embora o 
acúmulo seja mais pronunciado nas formas inorgânicas (Daroub et al., 2000). A redistribuição de $\mathrm{P}$ em diversas formas quando da fertilização também ocorre em solos cultivados sob sistema plantio direto (SPD), pois tem se observado a formação de uma camada de solo junto à superfície com alto teor de matéria orgânica e disponibilidade de nutrientes, inclusive P (Rheinheimer et al., 2003). Esse comportamento é conseqüência da adição consecutiva de fertilizantes na camada superficial do solo, ausência de revolvimento do solo e diminuição da taxa de erosão. A adsorção do $\mathrm{P}$ ocorre primeiramente nos sítios mais ávidos (de menor labilidade) e, posteriormente, o P remanescente é redistribuído em frações retidas com menor energia e de maior disponibilidade para as plantas (Rheinheimer \& Anghinoni, 2001). Adicionalmente, no SPD a manutenção dos resíduos culturais na superfície do solo reduz sua taxa de decomposição, aumentando o conteúdo de matéria orgânica e de Po. A maior atividade microbiana na camada superficial do solo sob SPD aumenta o estoque de Po armazenado na biomassa microbiana, o qual pode se tornar disponível via mineralização (Conte, 2001).

Rheinheimer (2000) estudou as formas de P em solos com diferentes teores de argila submetidos ao preparo convencional e ao sistema plantio direto e observou que no SPD houve aumento de $\mathrm{P}$ nas camadas superficiais, principalmente nas formas inorgânicas disponíveis e moderadamente disponíveis, permitindo a manutenção de teores de $\mathrm{P}$ na solução do solo mais elevados do que no preparo convencional. Contudo, em solos sob SPD, ainda não há como precisar a participação efetiva das formas de $\mathrm{P}$ na biodisponibilidade, pois, embora já tenham sido caracterizadas, pouco se sabe sobre a capacidade de dessorção dessas formas de $\mathrm{P}$ e sua atuação no tamponamento da solução do solo.

O objetivo do presente trabalho foi avaliar a biodisponibilidade de formas de $\mathrm{P}$ acumuladas em um solo cultivado sob sistema plantio direto com diferentes quantidades de $\mathrm{P}$ adicionado.

\section{MATERIAL E MÉTODOS}

Para realização deste estudo foram utilizadas amostras de solo coletadas no município de Santo Ângelo, Rio Grande do Sul. As amostras foram coletadas em janeiro de 2000, em um experimento com doses de P, instalado em 1994, em um Latossolo Vermelho distroférrico típico, conduzido sob sistema plantio direto. No momento da coleta, o solo apresentava as seguintes características: $706 \mathrm{~g} \mathrm{~kg}^{-1}$ de argila; $223 \mathrm{~g} \mathrm{~kg}^{-1}$ de silte; $71 \mathrm{~g} \mathrm{~kg}^{-1}$ de areia; $129 \mathrm{~g} \mathrm{~kg}^{-1}$ de Fe extraído por ditionito-citratobicarbonato; $70 \mathrm{~g} \mathrm{~kg}^{-1}$ de Fe extraído por oxalato de amônio; $17,2 \mathrm{~g} \mathrm{~kg}^{-1}$ de C orgânico; $\mathrm{pH}$ em água = 4,6; índice $\mathrm{SMP}=5,4 ; \mathrm{Al} \mathrm{Al}$ trocável = 0,85 $\mathrm{cmol}_{\mathrm{c}} \mathrm{kg}^{-1}$;
Ca trocável $=2,71 \mathrm{cmol}_{\mathrm{c}} \mathrm{kg}^{-1} ; \mathrm{Mg}$ trocável = $0,96 \mathrm{cmol}_{\mathrm{c}} \mathrm{kg}^{-1} ; \mathrm{K}$ disponível $=270 \mathrm{mg} \mathrm{kg}^{-1}$. Foram coletados $15 \mathrm{~kg}$ de solo da camada de $0-0,10 \mathrm{~m}$ de parcelas que receberam doses anuais de $0,30,60,90 \mathrm{e}$ $120 \mathrm{~kg} \mathrm{ha}^{-1}$ ano $^{-1} \mathrm{de}_{2} \mathrm{O}_{5}$, totalizando 0, 180, 360, 540 e $720 \mathrm{~kg} \mathrm{ha}{ }^{-1} \mathrm{de}_{2} \mathrm{O}_{5}$ aplicados até o momento da coleta. Os teores de $\mathrm{P}$ disponível por Mehlich-1 eram de 8,6; 11,$4 ; 13,3 ; 21,0$ e $30,0 \mathrm{mg} \mathrm{kg}^{-1}$, respectivamente, para as doses anuais de 0, 30, 60, 90 e $120 \mathrm{~kg} \mathrm{ha}^{-1}$ de $\mathrm{P}_{2} \mathrm{O}_{5}$.

Após a coleta, o solo foi seco ao ar, moído, tamisado em malha de 2,0 $\mathrm{mm}$ e acondicionado em vasos com capacidade de $2,0 \mathrm{~kg}$. O experimento foi realizado em casa de vegetação do Departamento de Solos da Universidade Federal de Santa Maria (UFSM), no período de 21/2/2000 a 25/1/2002, e as análises, no Laboratório de Química e Fertilidade do Solo do Departamento de Solos da UFSM.

O experimento constou de 15 cultivos sucessivos em casa de vegetação, e os tratamentos foram as cinco doses de $\mathrm{P}$ provindas do experimento a campo. $\mathrm{O}$ delineamento experimental utilizado foi o bifatorial inteiramente casualizado (fator $\mathrm{A}=$ cinco doses de $\mathrm{Pe}$ fator $\mathrm{B}=$ cultivos ou subdivisões temporais), com seis repetições.

As espécies cultivadas foram: milheto Pennisetum americanum (cultivo 1); aveia-preta Avena strigosa (cultivos 2, 3 e 4); milho-Zea mayz (cultivo 5); soja - Glycine max (cultivos 6, 7, 8, 9, 13, 14 e 15); crotalária - Crotalaria spectabilis (cultivo 10) e centeio - Secale cereale (cultivos 11 e 12). As sementes foram pré-germinadas em laboratório até a emissão da radícula e transplantadas de quatro (espécies de rápido crescimento) a 15 plantas (espécies de crescimento lento) para os vasos. Seguindo as recomendações da CFSRS/SC (1994), antes do primeiro e do quinto cultivo, todos os tratamentos receberam adição de $\mathrm{K}, \mathrm{Ca}, \mathrm{Mg}, \mathrm{S}, \mathrm{Zn}, \mathrm{B}, \mathrm{Cu}$ e Mo. No oitavo cultivo, foi adicionado calcário ao solo para manter o $\mathrm{pH}$ próximo a 6,0. Em cada cultivo sucessivo, as plantas foram cultivadas por períodos variáveis de 30 a 45 dias, sendo o corte realizado quando a taxa de crescimento vegetativo das plantas diminuía. Após cada colheita, o solo foi tamisado, seco em estufa e recolocado nos vasos para novo cultivo. Em cada cultivo foi determinada a produção de matéria seca da parte aérea e a das raízes. O P absorvido foi determinado na parte aérea e nas raízes após moagem da fitomassa em moinho tipo Willey e digestão com $\mathrm{H}_{2} \mathrm{SO}_{4}+\mathrm{H}_{2} \mathrm{O}_{2}$ (Tedesco et al., 1995).

Foram coletadas amostras de solo antes da realização dos cultivos e após três, seis, nove, doze e quinze cultivos, as quais foram submetidas ao fracionamento químico do $\mathrm{P}$ (Hedley et al., 1982), com as modificações propostas por Condron et al. (1985), descrito a seguir. Amostras de 0,5 g de solo foram submetidas à extração, seqüencialmente, com resina trocadora de ânions (placas AR 103 QDP 434), $\mathrm{NaHCO}_{3} 0,5 \mathrm{~mol} \mathrm{~L}^{-1}, \mathrm{NaOH} 0,1 \mathrm{~mol} \mathrm{~L}^{-1}, \mathrm{HCl}$ $1,0 \mathrm{~mol} \mathrm{~L}^{-1} \mathrm{e} \mathrm{NaOH} \mathrm{0,5} \mathrm{mol} \mathrm{L}^{-1}$. Após essas extrações, 
o solo remanescente foi seco em estufa e submetido à digestão com $\mathrm{H}_{2} \mathrm{SO}_{4}+\mathrm{H}_{2} \mathrm{O}_{2}+\mathrm{MgCl}_{2}$ (Brookes \& Powson, 1981). O Pi dos extratos alcalinos de $\mathrm{NaHCO}_{3}$ e $\mathrm{NaOH}$ foi analisado pelo método de Dick \& Tabatabai (1977). Nos extratos alcalinos foi determinado o P total por digestão com persulfato de amônio + ácido sulfúrico em autoclave (USEPA, 1971), sendo o Po obtido pela diferença entre $\mathrm{P}$ total e $\mathrm{P}$ inorgânico. $\mathrm{O}$ $\mathrm{P}$ dos extratos ácidos foi determinado segundo método de Murphy \& Riley (1962).

Neste trabalho, cada vez que foi realizada análise de fracionamento de $\mathrm{P}$, verificou-se a eficiência de extração deste elemento, comparando a soma das frações com os valores totais de $\mathrm{P}$ do solo. Para isso, foram somados os teores obtidos em todas as formas de $\mathrm{Pi}$ do fracionamento, incluindo o $\mathrm{P}$ residual $\left(\mathrm{Pi}_{\mathrm{RTA}}\right.$ $\left.+\mathrm{Pi}_{\text {bic }}+\mathrm{Pi}_{\text {hid }}+\mathrm{Pi}_{\text {hid } 0,5}+\mathrm{Pi}_{\mathrm{HCl}}+\mathrm{P}_{\text {res }}\right)$, e os valores foram comparados com o Pi total do solo determinado pelo método descrito em Olsen \& Sommers (1982) (dados não apresentados). Similarmente, foram somados os teores de todas as formas de Po do fracionamento $\left(\mathrm{Po}_{\text {bic }}+\mathrm{Po}_{\text {hid }}+\mathrm{Po}_{\text {hid }, 5}\right)$ e comparados com os resultados de Po total determinado por ignição (Olsen \& Sommers, 1982) (dados não apresentados). Os resultados mostraram que o fracionamento extraiu, em média, 78,1 \% do Pi total, 41,9\% do Po total e $68,5 \%$ do $\mathrm{P}$ total do solo. As correlações lineares entre os teores obtidos pelo fracionamento e pelo método de Olsen \& Sommers (1982) foram de 0,86, 0,62 e 0,89 para $\mathrm{Pi}$, Po e $\mathrm{P}$ total, respectivamente.

Os resultados de cada forma de $\mathrm{P}$ do fracionamento químico do solo foram submetidos à análise de variância, considerando as doses de $\mathrm{P}$ como fator principal e os cultivos como subdivisões temporais. Quando constatados efeitos significativos pela análise da variância, foram ajustadas equações de regressão para as doses de $\mathrm{P}$ em cada cultivo, e as diferenças nos teores de $\mathrm{P}$ entre os cultivos foram comparadas pelo teste DMS (diferença mínima significativa) a $5 \%$.

\section{RESULTADOS E DISCUSSÃO}

Os resultados mostram que os teores de $\mathrm{P}$ inorgânico extraídos pelo primeiro extrator $\left(\mathrm{Pi}_{\mathrm{RTA}}\right)$ foram crescentes com o aumento da dose de fertilizante aplicada no solo original, e os teores diminuíram em todos os tratamentos após os cultivos sucessivos sem reposição do $\mathrm{P}$ absorvido pelas plantas (Quadro 1). Em alguns tratamentos, foi observado, tanto na fração $\mathrm{Pi}_{\text {RTA }}$ como na $\mathrm{Pi}_{\text {bic }}$, aumento dos teores de $\mathrm{Pi}$ do terceiro para o sexto cultivo (Quadro 1) e, concomitantemente, houve diminuição dos teores de Po extraídos por bicarbonato $\left(\mathrm{Po}_{\mathrm{bic}}\right)$, indicando que a absorção de Pi pelas plantas desencadeou um processo de mineralização de parte do $\mathrm{Po}$, repondo o $\mathrm{Pi}$.

Os resultados mostraram depleção do $\mathrm{Pi}_{\text {bic }}$ com os cultivos, sendo mais evidente nos tratamentos com maior adição de $\mathrm{P}$ (540 e $720 \mathrm{~kg} \mathrm{ha}^{-1}$ de $\left.\mathrm{P}_{2} \mathrm{O}_{5}\right)$, indicando que nestes tratamentos havia $\mathrm{P}$ acumulado nessa forma e ele foi utilizado pelas plantas. Já nos outros tratamentos $\left(0,180\right.$ e $360 \mathrm{~kg} \mathrm{ha}^{-1}$ de $\left.\mathrm{P}_{2} \mathrm{O}_{5}\right)$, pela maior carência de $\mathrm{P}$, os teores iniciais e aqueles após 15 cultivos foram semelhantes, indicando que essa forma foi pouco utilizada devido aos baixos teores ou, ainda, que foi utilizada e reposta por formas de menor labilidade.

A partir do nono cultivo, foi observado significativo aumento nos teores de $\mathrm{Po}$ lábil $\left(\mathrm{Po}_{\mathrm{bic}}\right)$, que perdurou até o décimo quinto cultivo (Quadro 1). Isso provocou aumento dos teores totais nessa fração $\left(\mathrm{Pi}_{\text {bic }}+\mathrm{Po}_{\text {bic }}\right)$, mostrando que houve migração de $\mathrm{P}$ de outras frações para o $\mathrm{Po}_{\text {bic. }}$ Segundo Sharpley \& Smith (1985) e Chen et al. (2002), isso pode ocorrer devido à transformação de formas mais estáveis de Po em formas mais lábeis com o cultivo. No presente trabalho, a tamisagem do solo realizada após cada cultivo pode ter potencializado a extração de $\mathrm{P}_{\mathrm{o}_{\text {bic }}}$, pois ela poderia ter diminuído a agregação do solo e, conseqüentemente, a proteção física da matéria orgânica, possibilitando que o Po fosse extraído por soluções extratoras mais fracas.

A estreita relação dinâmica entre as frações $\mathrm{Pi}_{\mathrm{RTA}}$, $\mathrm{Pi}_{\text {bic }}$ e $\mathrm{Po}_{\text {bic }}$ e sua sensibilidade à absorção de $\mathrm{P}$ pelas plantas sugerem que esses compartimentos são sensíveis à diminuição do conteúdo de $\mathrm{P}$ na solução do solo e que, juntos, compõem o chamado P lábil do solo, como defendido por Hedley et al. (1982), Tiessen et al. (1984), Beck \& Sanches (1984), Sharpley \& Smith (1985), Stewart \& Tiessen (1987), Guo \& Yost (1998) e Henriquez \& Killorn (2005). Devido à grande importância na disponibilidade para as plantas das frações inorgânicas e orgânicas extraídas por bicarbonato, Adepetu \& Corey (1979), Tiessen et al. (1984), Sharpley et al. (1987) e Maroko et al. (1999) sugerem que essas formas sejam incluídas como indicadoras de disponibilidade de $\mathrm{P}$ em sistemas com baixa disponibilidade e baixa adição de fertilizantes.

No fracionamento de Hedley, após as extrações com RTA e $\mathrm{NaHCO}_{3}$ o solo é submetido à extração com $\mathrm{NaOH} 0,1 \mathrm{~mol} \mathrm{~L}^{-1}$ e $\mathrm{NaOH} 0,5 \mathrm{~mol} \mathrm{~L}^{-1}$, sendo dessorvidas formas inorgânicas e orgânicas de $\mathrm{P}$ de menor labilidade, pois, como as formas inorgânicas lábeis já foram retiradas pelos extratores anteriores, as formas de $\mathrm{P}$ extraídas pelas soluções de $\mathrm{NaOH}$ são de labilidade intermediária. Os teores de $\mathrm{P}$ extraído com NaOH 0,1 mol L-1 $\left(\mathrm{Pi}_{\text {hid }}\right.$ e $\left.\mathrm{Po}_{\text {hid }}\right)$ são apresentados no quadro 1 , onde se pode observar que os teores de $\mathrm{Pi}$ aumentaram até o décimo segundo cultivo, diminuindo apenas após o décimo quinto. Para o Pi extraído com $\mathrm{NaOH} \mathrm{0,5} \mathrm{mol} \mathrm{L-1} \mathrm{(Quadro} \mathrm{2),} \mathrm{foi} \mathrm{observado} \mathrm{aumento}$ de teores até o décimo quinto cultivo. Para as frações orgânicas $\left(\mathrm{Po}_{\text {hid }}\right.$ e $\left.\mathrm{P}_{\text {hid0,5 }}\right)$, ocorreu diminuição dos teores de Po após os cultivos, indicando que a diminuição dos teores disponíveis provocou a mineralização de Po, como observado por Gatiboni et al. (2005). Como não houve aumento nas frações mais lábeis $\left(\mathrm{Pi}_{\mathrm{RTA}}\right.$ e $\left.\mathrm{Pi}_{\text {bic }}\right)$, provavelmente o $\mathrm{P}$ proveniente da mineralização do Po extraído por hidróxido tenha 
Quadro 1. Teores de fósforo inorgânico (Pi) e orgânico (Po) extraídos seqüencialmente por $\mathrm{RTA}^{\mathrm{N}} \mathrm{NaHCO}_{3}$ $0,5 \mathrm{~mol} \mathrm{~L}^{-1} \mathrm{e} \mathrm{NaOH} 0,1 \mathrm{~mol} \mathrm{~L}^{-1} \mathrm{em}$ amostras de um Latossolo Vermelho distroférrico típico com diferentes históricos de adubação, coletadas antes e após a realização de cultivos sucessivos em casa de vegetação

\begin{tabular}{|c|c|c|c|c|c|c|c|}
\hline \multirow{2}{*}{ Extrator de $\mathbf{P}$} & \multirow{2}{*}{ Cultivo } & \multicolumn{5}{|c|}{ Dose de $\mathrm{P}_{2} \mathrm{O}_{5}\left(\mathrm{~kg} \mathrm{ha}^{-1}\right)$} & \multirow{2}{*}{ Equação } \\
\hline & & 0 & 180 & 360 & 540 & 720 & \\
\hline \multirow{7}{*}{$\begin{array}{c}\text { RTA } \\
\left(\operatorname{Pi}_{\text {RTA }}\right)\end{array}$} & $0^{(1)}$ & $8,6 \mathrm{a}^{(2)}$ & $11,4 \mathrm{a}$ & $13,3 \mathrm{a}$ & $21,0 \mathrm{a}$ & $30,0 \mathrm{a}$ & $\hat{\mathrm{Y}}=8,939+0,0005 \mathrm{X}+0,00004 \mathrm{X}^{2}\left(\mathrm{R}^{2}=0,99\right)$ \\
\hline & 3 & $5,0 \mathrm{c}$ & $6,6 \mathrm{bc}$ & $7,4 \mathrm{~b}$ & $12,6 \mathrm{c}$ & $19,3 \mathrm{~b}$ & $\hat{\mathrm{Y}}=5,340+0,0040 \mathrm{X}+0,00003 \mathrm{X}^{2}\left(\mathrm{R}^{2}=0,99\right)$ \\
\hline & 6 & $5,7 \mathrm{~b}$ & $7,3 \mathrm{~b}$ & $8,7 \mathrm{~b}$ & $15,6 \mathrm{~b}$ & $21,2 \mathrm{~b}$ & $\hat{\mathrm{Y}}=5,797+0,0004 \mathrm{X}+0,00003 \mathrm{X}^{2}\left(\mathrm{R}^{2}=0,99\right)$ \\
\hline & 9 & $5,6 \mathrm{bc}$ & $7,7 \mathrm{~b}$ & $8,8 \mathrm{~b}$ & $14,7 \mathrm{~b}$ & $18,8 \mathrm{~b}$ & $\hat{\mathrm{Y}}=5,645+0,0049 \mathrm{X}+0,00002 \mathrm{X}^{2}\left(\mathrm{R}^{2}=0,98\right)$ \\
\hline & 12 & $3,8 \mathrm{~d}$ & $5,7 \mathrm{c}$ & $7,3 \mathrm{~b}$ & $12,4 \mathrm{~cd}$ & $14,0 \mathrm{c}$ & $\hat{\mathrm{Y}}=3,234+0,0151 \mathrm{X}\left(\mathrm{R}^{2}=0,96\right)$ \\
\hline & 15 & $4,9 \mathrm{c}$ & $6,0 \mathrm{c}$ & $7,0 \mathrm{~b}$ & $10,8 \mathrm{~d}$ & $11,8 \mathrm{c}$ & $\hat{\mathrm{Y}}=4,378+0,0104 \mathrm{X}\left(\mathrm{R}^{2}=0,94\right)$ \\
\hline & $\mathrm{CV}(\%)$ & & & & & 9,05 & \\
\hline \multirow{7}{*}{$\begin{array}{c}\mathrm{NaHCO}_{3} \\
0,5 \mathrm{~mol} \mathrm{~L}^{-1} \\
\left(\mathrm{Pi}_{\text {bic }}\right)\end{array}$} & 0 & $4,7 \mathrm{~b}$ & $6,8 \mathrm{a}$ & $7,2 \mathrm{~b}$ & $15,3 \mathrm{a}$ & $21,1 \mathrm{a}$ & $\hat{\mathrm{Y}}=4,932+0,0010 \mathrm{X}+0,00003 \mathrm{X}^{2}\left(\mathrm{R}^{2}=0,97\right)$ \\
\hline & 3 & $5,1 \mathrm{ab}$ & $5,9 \mathrm{bc}$ & $6,6 \mathrm{bc}$ & $12,5 \mathrm{~b}$ & $17,5 \mathrm{~b}$ & $\hat{\mathrm{Y}}=5,151-0,0039 \mathrm{X}+0,00003 \mathrm{X}^{2}\left(\mathrm{R}^{2}=0,98\right)$ \\
\hline & 6 & 5,9 a & $7,1 \mathrm{a}$ & $7,1 \mathrm{bc}$ & $12,2 \mathrm{~b}$ & $16,2 \mathrm{~b}$ & $\hat{\mathrm{Y}}=6,037-0,0023 \mathrm{X}+0,00002 \mathrm{X}^{2}\left(\mathrm{R}^{2}=0,97\right)$ \\
\hline & 9 & $5,3 \mathrm{ab}$ & $6,9 \mathrm{a}$ & $7,3 \mathrm{~b}$ & $11,3 \mathrm{bc}$ & $14,0 \mathrm{c}$ & $\hat{\mathrm{Y}}=5,453+0,0027 \mathrm{X}+0,00001 \mathrm{X}^{2}\left(\mathrm{R}^{2}=0,97\right)$ \\
\hline & 12 & $5,8 \mathrm{a}$ & $6,7 \mathrm{ab}$ & $8,4 \mathrm{a}$ & $10,6 \mathrm{c}$ & $11,8 \mathrm{~d}$ & $\hat{\mathrm{Y}}=5,506+0,0088 \mathrm{X}\left(\mathrm{R}^{2}=0,98\right)$ \\
\hline & 15 & $5,6 \mathrm{ab}$ & $5,8 \mathrm{c}$ & $6,1 \mathrm{c}$ & $8,5 \mathrm{~d}$ & $9,6 \mathrm{e}$ & $\hat{\mathrm{Y}}=5,546-0,0003 \mathrm{X}+0,000009 \mathrm{X}^{2}\left(\mathrm{R}^{2}=0,95\right)$ \\
\hline & $\mathrm{CV}(\%)$ & & & & & 9,05 & \\
\hline \multirow{7}{*}{$\begin{array}{c}\mathrm{NaHCO}_{3} \\
0,5 \mathrm{~mol} \mathrm{~L}^{-1} \\
\text { (Pobic) }\end{array}$} & 0 & $19,8 \mathrm{~b}$ & $19,5 \mathrm{~cd}$ & $19,7 \mathrm{c}$ & $20,4 \mathrm{c}$ & $18,6 \mathrm{de}$ & $\mathrm{NS}^{(3)}$ \\
\hline & 3 & $20,7 \mathrm{~b}$ & $21,2 \mathrm{bc}$ & $19,5 \mathrm{c}$ & $19,6 \mathrm{c}$ & $21,6 \mathrm{~cd}$ & NS \\
\hline & 6 & $17,0 \mathrm{c}$ & $17,0 \mathrm{~d}$ & $15,8 \mathrm{~d}$ & $16,5 \mathrm{~d}$ & $16,7 \mathrm{e}$ & NS \\
\hline & 9 & $24,1 \mathrm{a}$ & $23,6 \mathrm{ab}$ & $23,8 \mathrm{~b}$ & $27,3 \mathrm{ab}$ & $24,8 \mathrm{bc}$ & NS \\
\hline & 12 & 24,4 a & $25,2 \mathrm{a}$ & $23,8 \mathrm{~b}$ & $25,4 \mathrm{~b}$ & $27,2 \mathrm{ab}$ & NS \\
\hline & 15 & 25,9 a & 25,4 a & 28,3 a & $28,8 \mathrm{a}$ & $30,0 \mathrm{a}$ & $\hat{\mathrm{Y}}=25,372+0,0064 \mathrm{X}\left(\mathrm{R}^{2}=0,87\right)$ \\
\hline & CV (\%) & & & & & 8,23 & \\
\hline \multirow{7}{*}{$\begin{array}{c}\mathrm{NaOH} \\
0,1 \mathrm{~mol} \mathrm{~L}^{-1} \\
\left(\mathrm{Pi}_{\text {hido,1 }}\right)\end{array}$} & 0 & $57,3 \mathrm{~d}$ & $57,7 \mathrm{~d}$ & $60,6 \mathrm{~d}$ & $91,6 \mathrm{c}$ & $98,7 \mathrm{~d}$ & $\hat{\mathrm{Y}}=55,739-0,0008 \mathrm{X}+0,00009 \mathrm{X}^{2}\left(\mathrm{R}^{2}=0,91\right)$ \\
\hline & 3 & $58,9 \mathrm{~d}$ & $60,1 \mathrm{~d}$ & $61,4 \mathrm{~d}$ & $89,4 \mathrm{c}$ & $112,6 \mathrm{c}$ & $\hat{\mathrm{Y}}=59,271-0,0364 \mathrm{X}+0,00020 \mathrm{X}^{2}\left(\mathrm{R}^{2}=0,98\right)$ \\
\hline & 6 & $66,6 \mathrm{c}$ & $71,5 \mathrm{c}$ & $68,7 \mathrm{c}$ & $97,0 \mathrm{c}$ & $116,9 \mathrm{c}$ & $\hat{\mathrm{Y}}=67,651-0,0270 \mathrm{X}+0,00010 \mathrm{X}^{2}\left(\mathrm{R}^{2}=0,96\right)$ \\
\hline & 9 & 104,9 a & 115,0 a & 110,6 a & 146,7 a & $164,1 \mathrm{a}$ & $\hat{\mathrm{Y}}=106,10-0,0038 \mathrm{X}+0,00010 \mathrm{X}^{2}\left(\mathrm{R}^{2}=0,93\right)$ \\
\hline & 12 & $104,0 \mathrm{a}$ & $112,0 \mathrm{a}$ & $113,5 \mathrm{a}$ & 148,8 a & $162,6 \mathrm{a}$ & $\hat{\mathrm{Y}}=103,86+0,0137 \mathrm{X}+0,00010 \mathrm{X}^{2}\left(\mathrm{R}^{2}=0,94\right)$ \\
\hline & 15 & $81,6 \mathrm{~b}$ & $93,7 \mathrm{~b}$ & $93,5 \mathrm{~b}$ & $117,5 \mathrm{~b}$ & $131,3 \mathrm{~b}$ & $\hat{\mathrm{Y}}=82,819+0,0245 \mathrm{X}+0,00006 \mathrm{X}^{2}\left(\mathrm{R}^{2}=0,96\right)$ \\
\hline & $\mathrm{CV}(\%)$ & & & & & 4,71 & \\
\hline \multirow{7}{*}{$\begin{array}{c}\mathrm{NaOH} \\
0,1 \mathrm{~mol} \mathrm{~L}^{-1} \\
(\text { Pohido,1 })\end{array}$} & 0 & 93,2 a & $106,0 \mathrm{a}$ & $103,6 \mathrm{a}$ & $115,8 \mathrm{a}$ & $128,1 \mathrm{a}$ & $\hat{\mathrm{Y}}=93,414+0,0442 \mathrm{X}\left(\mathrm{R}^{2}=0,91\right)$ \\
\hline & 3 & $76,4 \mathrm{~b}$ & $87,1 \mathrm{~b}$ & $85,4 \mathrm{~b}$ & $91,6 \mathrm{~b}$ & $98,0 \mathrm{ab}$ & $\hat{\mathrm{Y}}=78,168+0,0264 \mathrm{X}\left(\mathrm{R}^{2}=0,89\right)$ \\
\hline & 6 & 92,5 a & $99,2 \mathrm{ab}$ & 104,9 a & $87,8 \mathrm{~b}$ & $89,2 \mathrm{bc}$ & NS \\
\hline & 9 & $48,0 \mathrm{c}$ & $55,5 \mathrm{c}$ & $51,8 \mathrm{c}$ & $53,9 \mathrm{c}$ & $52,7 \mathrm{~d}$ & NS \\
\hline & 12 & $50,1 \mathrm{c}$ & $56,4 \mathrm{c}$ & $51,1 \mathrm{c}$ & $54,7 \mathrm{c}$ & $55,5 \mathrm{~d}$ & NS \\
\hline & 15 & $46,6 \mathrm{c}$ & $50,9 \mathrm{c}$ & $51,0 \mathrm{c}$ & $66,7 \mathrm{c}$ & $63,3 \mathrm{~cd}$ & $\hat{\mathrm{Y}}=45,81+0,0274 \mathrm{X}\left(\mathrm{R}^{2}=0,79\right)$ \\
\hline & $\mathrm{CV}(\%)$ & & & & & 13,52 & \\
\hline
\end{tabular}

(1) Espécies cultivadas: milheto - Pennisetum americanum (cultivo 1); aveia-preta - Avena strigosa (cultivos 2, 3 e 4); milho - Zea mayz (cultivo 5); soja - Glycine max (cultivos 6, 7, 8, 9, 13, 14 e 15); crotalária - Crotalaria spectabilis (cultivo 10); centeio - Secale cereale (cultivos 11 e 12). ${ }^{(2)}$ Médias seguidas pela mesma letra, na coluna, não diferem entre si pelo teste DMS ( $\left.p<0,05\right) .{ }^{(3)}$ NS $=$ não-significativo $(\mathrm{p}<0,05)$.

sido parte absorvido pelas plantas e parte readsorvido ao solo em outras formas, já que houve aumento nas frações $\mathrm{Pi}_{\text {hid }}$ e $\mathrm{Pi}_{\text {hid } 0,5}$.

Do sexto para o nono cultivo ocorreu grande depleção do $\mathrm{Po}_{\text {hid }}$, sendo parte deste, provavelmente, transformada em formas orgânicas mais lábeis $\left(\mathrm{Po}_{\text {bic }}\right)$ e parte transformada em Pi (mineralizado). A mineralização de Po deve ter provocado a readsorção na fração $\mathrm{Pi}_{\text {hid }}$, que apresentou grande aumento de teores. Esse fenômeno pode ser explicado pela adição de calcário ao solo no oitavo cultivo, que, provavelmente, assim como observado por Beck \& Sanches (1994), aumentou a atividade microbiana do solo, provocando a mineralização de Po.

Após 15 cultivos, houve alta depleção do $\mathrm{Pi}_{\text {hid }} \mathrm{e}$ preservação do $\mathrm{Po}_{\text {hid }}$. Com isso, os teores totais desta 


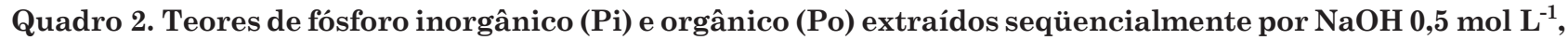
HCl $1 \mathrm{~mol} \mathrm{~L}^{-1}$ e fósforo remanescente no solo estimado por digestão ácida em amostras de um Latossolo Vermelho distroférrico típico com diferentes históricos de adubação, coletadas antes e após a realização de cultivos sucessivos em casa de vegetação

\begin{tabular}{|c|c|c|c|c|c|c|c|}
\hline \multirow{2}{*}{ Extrator de $\mathbf{P}$} & \multirow{2}{*}{ Cultivo } & \multicolumn{5}{|c|}{ Dose de $\mathrm{P}_{2} \mathrm{O}_{5}\left(\mathrm{~kg} \mathrm{ha}^{-1}\right)$} & \multirow{2}{*}{ Equação } \\
\hline & & $\mathbf{0}$ & 180 & 360 & 540 & 720 & \\
\hline \multirow{6}{*}{$\begin{array}{c}\mathrm{NaOH} \\
0,5 \mathrm{~mol} \mathrm{~L}^{-1} \\
(\text { Pihid } 0,5))\end{array}$} & $0^{(1)}$ & $73,5 \mathrm{~b}^{(2)}$ & $74,9 \mathrm{~b}$ & $89,6 \mathrm{c}$ & $85,9 \mathrm{c}$ & $92,1 \mathrm{~b}$ & $\hat{\mathrm{Y}}=73,582+0,0267 \mathrm{X}\left(\mathrm{R}^{2}=0,80\right)$ \\
\hline & 3 & $77,5 \mathrm{~b}$ & $85,7 \mathrm{~b}$ & $88,5 \mathrm{c}$ & $86,2 \mathrm{c}$ & $98,8 \mathrm{~b}$ & $\hat{\mathrm{Y}}=78,758+0,0239 \mathrm{X}\left(\mathrm{R}^{2}=0,79\right)$ \\
\hline & 6 & $73,1 \mathrm{~b}$ & $86,1 \mathrm{~b}$ & $76,4 \mathrm{~d}$ & $82,9 \mathrm{c}$ & $96,0 \mathrm{~b}$ & $\hat{\mathrm{Y}}=74,390+0,0236 \mathrm{X}\left(\mathrm{R}^{2}=0,56\right)$ \\
\hline & 9 & 116,9 a & $121,3 \mathrm{a}$ & $123,1 \mathrm{~b}$ & $132,1 \mathrm{~b}$ & $134,1 \mathrm{a}$ & $\hat{\mathrm{Y}}=116,47+0,0251 \mathrm{X}\left(\mathrm{R}^{2}=0,95\right)$ \\
\hline & 12 & 118,4 a & $127,1 \mathrm{a}$ & $129,0 \mathrm{ab}$ & $132,7 \mathrm{~b}$ & $136,0 \mathrm{a}$ & $\hat{\mathrm{Y}}=120,47+0,0227 \mathrm{X}\left(\mathrm{R}^{2}=0,93\right)$ \\
\hline & $\begin{array}{c}15 \\
\mathrm{CV}(\%)\end{array}$ & 121,8 a & $131,5 \mathrm{a}$ & $138,4 \mathrm{a}$ & $147,4 \mathrm{a}$ & $\begin{array}{c}147,9 \mathrm{a} \\
7,57\end{array}$ & $\hat{\mathrm{Y}}=123,77+0,0379 \mathrm{X}\left(\mathrm{R}^{2}=0,95\right)$ \\
\hline \multirow{7}{*}{$\begin{array}{c}\mathrm{NaOH} \\
0,5 \mathrm{~mol} \mathrm{~L}^{-1} \\
(\text { Pohid } 0,5)\end{array}$} & 0 & $34,4 \mathrm{ab}$ & $45,9 \mathrm{a}$ & 35,2 a & 42,9 a & $34,8 \mathrm{a}$ & $\mathrm{NS}^{(3)}$ \\
\hline & 3 & $27,3 \mathrm{~b}$ & 38,5 a & $30,7 \mathrm{ab}$ & $33,6 \mathrm{abc}$ & $30,3 \mathrm{ab}$ & NS \\
\hline & 6 & $38,2 \mathrm{a}$ & 34,7 a & $40,3 \mathrm{a}$ & $37,6 \mathrm{ab}$ & $35,9 \mathrm{ab}$ & NS \\
\hline & 9 & $12,0 \mathrm{c}$ & $14,1 \mathrm{~b}$ & $15,0 \mathrm{c}$ & $19,4 \mathrm{c}$ & $15,9 \mathrm{~b}$ & NS \\
\hline & 12 & $11,7 \mathrm{c}$ & $17,9 \mathrm{~b}$ & $15,9 \mathrm{c}$ & $23,8 \mathrm{c}$ & $21,3 \mathrm{ab}$ & $\hat{\mathrm{Y}}=13,086+0,0140 \mathrm{X}\left(\mathrm{R}^{2}=0,71\right)$ \\
\hline & 15 & $12,5 \mathrm{c}$ & $16,2 \mathrm{~b}$ & $16,3 \mathrm{bc}$ & $20,2 \mathrm{c}$ & $19,7 \mathrm{ab}$ & $\hat{\mathrm{Y}}=13,31+0,0102 \mathrm{X}\left(\mathrm{R}^{2}=0,87\right)$ \\
\hline & $\mathrm{CV}(\%)$ & & & & & 32,17 & \\
\hline \multirow{7}{*}{$\begin{array}{c}\mathrm{HCl} \\
1,0 \mathrm{~mol} \mathrm{~L}^{-1} \\
\left(\mathrm{Pi}_{\mathrm{HCl}}\right)\end{array}$} & 0 & $7,8 \mathrm{bc}$ & $8,8 \mathrm{c}$ & $9,2 \mathrm{c}$ & $11,0 \mathrm{c}$ & $11,4 \mathrm{~d}$ & $\hat{\mathrm{Y}}=7,756+0,0052 \mathrm{X}\left(\mathrm{R}^{2}=0,96\right)$ \\
\hline & 3 & $7,7 \mathrm{c}$ & $9,3 \mathrm{bc}$ & $9,1 \mathrm{c}$ & $10,8 \mathrm{c}$ & $12,5 \mathrm{c}$ & $\hat{\mathrm{Y}}=7,929+0,0029 \mathrm{X}+0,000005 \mathrm{X}^{2}\left(\mathrm{R}^{2}=0,95\right)$ \\
\hline & 6 & $8,4 \mathrm{bc}$ & $10,4 \mathrm{~b}$ & $10,1 \mathrm{~b}$ & $12,4 \mathrm{~b}$ & $12,3 \mathrm{~cd}$ & $\hat{\mathrm{Y}}=8,748+0,0054 \mathrm{X}\left(\mathrm{R}^{2}=0,96\right)$ \\
\hline & 9 & $12,0 \mathrm{a}$ & $13,4 \mathrm{a}$ & $12,9 \mathrm{a}$ & $14,7 \mathrm{a}$ & $15,3 \mathrm{a}$ & $\hat{\mathrm{Y}}=12,12+0,0043 \mathrm{X}\left(\mathrm{R}^{2}=0,86\right)$ \\
\hline & 12 & $11,8 \mathrm{a}$ & $13,3 \mathrm{a}$ & $12,8 \mathrm{a}$ & $14,2 \mathrm{a}$ & $13,8 \mathrm{~b}$ & $\hat{\mathrm{Y}}=12,20+0,0028 \mathrm{X}\left(\mathrm{R}^{2}=0,70\right)$ \\
\hline & 15 & $8,5 \mathrm{~b}$ & $10,1 \mathrm{bc}$ & $10,2 \mathrm{~b}$ & $11,7 \mathrm{bc}$ & $11,7 \mathrm{~cd}$ & $\hat{\mathrm{Y}}=8,994+0,0041 \mathrm{X}\left(\mathrm{R}^{2}=0,90\right)$ \\
\hline & $\mathrm{CV}(\%)$ & & & & & 5,27 & \\
\hline \multirow{6}{*}{$\begin{array}{c}\mathrm{H}_{2} \mathrm{SO}_{4}+\mathrm{H}_{2} \mathrm{O}_{2} \\
+\mathrm{MgCl}_{2} \\
\left(\mathrm{P}_{\text {residual }}\right)\end{array}$} & 0 & 425,0 a & 460,0 a & 449,0 a & 430,0 a & $435,0 \mathrm{a}$ & NS \\
\hline & 3 & 421,0 a & $434,0 \mathrm{ab}$ & $454,0 \mathrm{a}$ & 427,0 a & $437,0 \mathrm{a}$ & NS \\
\hline & 6 & $403,0 \mathrm{ab}$ & $414,0 \mathrm{bc}$ & $423,0 \mathrm{ab}$ & 425,0 a & $412,0 \mathrm{a}$ & NS \\
\hline & 9 & $404,0 \mathrm{ab}$ & $428,0 \mathrm{ab}$ & $425,0 \mathrm{ab}$ & $426,0 \mathrm{a}$ & 409,0 a & $\hat{\mathrm{Y}}=405,62+0,1296 \mathrm{X}-0,00020 \mathrm{X}^{2}\left(\mathrm{R}^{2}=0,90\right)$ \\
\hline & 12 & $370,0 \mathrm{c}$ & $382,0 \mathrm{~cd}$ & $381,0 \mathrm{bc}$ & 394,0 a & $364,0 \mathrm{~b}$ & NS \\
\hline & $\begin{array}{c}15 \\
\text { CV (\%) }\end{array}$ & $334,0 \mathrm{c}$ & $366,0 \mathrm{~d}$ & $350,0 \mathrm{c}$ & $298,0 \mathrm{~b}$ & $\begin{array}{c}296,0 \mathrm{c} \\
5,87\end{array}$ & $\hat{\mathrm{Y}}=343,30+0,0826 \mathrm{X}-0,00020 \mathrm{X}^{2}\left(\mathrm{R}^{2}=0,73\right)$ \\
\hline
\end{tabular}

(1) Espécies cultivadas: milheto - Pennisetum americanum (cultivo 1); aveia-preta - Avena strigosa (cultivos 2, 3 e 4); milho - Zea mayz (cultivo 5); soja - Glycine $\max ($ cultivos 6, 7, 8, 9, 13, 14 e 15); crotalária - Crotalaria spectabilis (cultivo 10); centeio - Secale cereale (cultivos 11 e 12). ${ }^{(2)}$ Médias seguidas pela mesma letra, na coluna, não diferem entre si pelo teste DMS (p < 0,05$)$. ${ }^{(3)}$ NS $=$ não-significativo $(\mathrm{p}<0,05)$.

forma $(\mathrm{Pi}+\mathrm{Po})$ também diminuíram, indicando que o $\mathrm{Pi}_{\text {hid }}$ atuou como fonte de $\mathrm{P}$, repondo a solução do solo quando da absorção das plantas.

Pode-se observar que os teores de $\mathrm{Pi}_{\text {hid } 0,5}$ e $\mathrm{Po}_{\text {hid } 0,5}$ não foram modificados expressivamente até o sexto cultivo, mostrando que estas formas foram pouco participativas quando a disponibilidade das formas mais lábeis era maior (Quadro 2). Do sexto para o nono cultivo houve aumento do $\mathrm{Pi}_{\text {hid } 0,5}$ e redução do $\mathrm{Po}_{\text {hid0,5 }}$, indicando que a adição de calcário provocou a mineralização do Po ou sua transformação em formas orgânicas mais lábeis. As duas possibilidades devem ter ocorrido, pois houve aumento concomitante nas formas $\mathrm{Pi}_{\text {hid }}, \mathrm{Pi}_{\text {hid } 0,5}$ e $\mathrm{Po}_{\text {bic }}$, conforme relatado anteriormente. $\mathrm{O}$ comportamento dessa fração $\left(\mathrm{Pi}_{\text {hid0,5}}\right)$ perante o cultivo do solo mostra que ela é similar à fração $\mathrm{NaOH} 0,1 \mathrm{~mol} \mathrm{~L}^{-1}$, mas, devido à sua maior interação com o solo, seus efeitos são observados mais tardiamente, já que, enquanto para o $\mathrm{NaOH}$ $0,1 \mathrm{~mol} \mathrm{~L}^{-1}$ houve diminuição de Po no terceiro cultivo sucessivo, para o $\mathrm{NaOH} 0,5 \mathrm{~mol} \mathrm{~L}^{-1}$ isso ocorreu apenas no nono cultivo.

A partir do nono cultivo, os teores totais $(\mathrm{Pi}+\mathrm{Po})$ extraídos por $\mathrm{NaOH} 0,5 \mathrm{~mol} \mathrm{~L}^{-1}$ aumentaram, e a fonte do $\mathrm{P}$ provavelmente é a fração residual, ou seja, o $\mathrm{Po}$ e Pi que não foram extraídos por nenhum dos primeiros 
cinco extratores do fracionamento. Como isso ocorreu após a aplicação de calcário, há possibilidade de esse $\mathrm{P}$ ser proveniente de uma ou da combinação dos seguintes mecanismos observados por Beck \& Sanches (1994): aumento da mineralização do Po da fração residual pelo aumento da atividade microbiana do solo com o aumento do pH; e diminuição da adsorção do fosfato com os colóides em virtude da competição do Pi com os íons hidroxila gerados na reação do calcário.

Ao extrator $\mathrm{HCl} 1 \mathrm{~mol} \mathrm{~L}-1$, utilizado após o $\mathrm{NaOH}$ $0,1 \mathrm{~mol} \mathrm{~L}^{-1}$, é atribuída a capacidade de dessorção de formas inorgânicas de $\mathrm{P}$ associadas ao Ca (Cross \& Schlesinger, 1995). Os teores estimados por esse extrator $\left(\mathrm{Pi}_{\mathrm{HCl}}\right)$ foram crescentes com o aumento da dose de $\mathrm{P}$ (Quadro 3). Os teores de $\mathrm{Pi}_{\mathrm{HCl}}$ aumentaram até o décimo segundo cultivo, atuando como dreno de $\mathrm{P}$ do sistema. $\mathrm{O}$ acúmulo mais expressivo nessa fração ocorreu após a aplicação de calcário, indicando que houve neoformação de fosfatos de Ca pelo aumento da atividade deste elemento na solução, conforme constatado por Rheinheimer (2000) e observado por Guo \& Yost (1998). Apenas após 15 cultivos foi observada pequena depleção do $\mathrm{Pi}_{\mathrm{HCl}}$. Contudo, como os teores dessa fração são relativamente pequenos em relação às outras formas, é de pouca importância na disponibilidade às plantas em solos intemperizados, como o utilizado neste experimento.

A fração $\mathrm{P}$ residual do fracionamento $\left(\mathrm{P}_{\text {res }}\right)$, obtida pela digestão ácida do solo, é constituída pelo $\mathrm{P}$ que não foi extraído pelos extratores seletivos do fracionamento de Hedley e é composta por formas de $\mathrm{Pi}$ e de Po recalcitrantes. Os teores de P nesta forma foram muito altos em relação às outras formas do fracionamento (Quadro 2), indicando que este solo é altamente sortivo e que grande parte do $\mathrm{P}$ se encontra em formas de alta energia de ligação com os colóides do solo, como observado também por Rheinheimer (2000) e Conte (2001) nos solos coletados da mesma unidade de mapeamento, no município de Santo Ângelo, Rio Grande do Sul.

$\mathrm{O} \mathrm{P}_{\text {res }}$ não foi aumentado pela adição de doses de fertilizantes (Quadro 2), indicando que o $\mathrm{P}$ adicionado é acumulado preferencialmente nas frações de maior labilidade. $\mathrm{O} \mathrm{P}_{\text {res }}$ não apresentou modificações expressivas até o nono cultivo, mas sofreu grande depleção após 12 e 15 cultivos. Isso mostra que o $\mathrm{P}_{\text {res }}$ foi preservado, enquanto as formas de labilidade intermediária tinham capacidade de repor as mais lábeis, indicando que, em sistemas com deficiência de $\mathrm{P}$ expressiva, todas as formas de $\mathrm{P}$ são participativas na reposição desse elemento da solução do solo, inclusive o $\mathrm{P}_{\text {res }}$, como observado por Guo \& Yost (1998).

Os resultados do fracionamento mostram que em sistemas com deficiência de $\mathrm{P}$ a absorção pelas culturas provoca o desencadeamento de efeito cascata de reposição do $\mathrm{P}$ lábil, primeiramente pelas formas de labilidade intermediária e, posteriormente, pelas formas de baixa labilidade. Isso indica que todas as formas de $\mathrm{P}$ podem ser disponibilizadas às plantas, porém, quanto maior a interação do $\mathrm{P}$ com os colóides do solo, menor é a velocidade de reposição das formas lábeis. Nesse sentido, a dinâmica das formas de $\mathrm{P}$ mostra que provavelmente a taxa de liberação deste elemento pelas formas menos lábeis não seja suficiente para manter o crescimento de cultivos comerciais, mas é, provavelmente, o mecanismo que sustenta o crescimento das plantas em ecossistemas naturais.

A quantidade de $\mathrm{P}$ absorvido pelas plantas (parte aérea + raízes) nos 15 cultivos mostrou que a absorção foi crescente com o nível de $\mathrm{P}$ acumulado no solo pelas adubações antecedentes (Quadro 3). Neste quadro são mostrados também os dados sobre a diferença do somatório de $\mathrm{P}$ das formas do fracionamento entre o solo inicial e aquele após os 15 cultivos $(\Delta \mathrm{P}$ fracionamento). Os resultados mostram que o fracionamento não foi hábil em detectar diferenças de absorção de $\mathrm{P}$ nas três primeiras doses de adubação estudadas $\left(0,180\right.$ e $360 \mathrm{~kg} \mathrm{ha}^{-1}$ de $\left.\mathrm{P}_{2} \mathrm{O}_{5}\right)$, permanecendo o $\Delta \mathrm{P}$ fracionamento na faixa dos $80 \mathrm{mg} \mathrm{kg}^{-1}$. Para as doses de 540 e $720 \mathrm{~kg} \mathrm{ha}^{-1}$ de $\mathrm{P}_{2} \mathrm{O}_{5}$, as diferenças entre os teores iniciais e finais foram mais distintas $\left(125,1\right.$ e $149,9 \mathrm{mg} \mathrm{kg}^{-1}$, respectivamente).

$\mathrm{O} \Delta \mathrm{P}$ também foi calculado separando-se as formas inorgânicas $\left(\Delta \mathrm{Pi}\right.$ fracionamento $=\mathrm{Pi}_{\mathrm{RTA}}+\mathrm{Pi}_{\text {bic }}+$ $\left.\mathrm{Pi}_{\text {hid }}+\mathrm{Pi}_{\mathrm{HCl}}+\mathrm{Pi}_{\text {hid } 0,5}+\mathrm{P}_{\text {res }}\right)$ e orgânicas $(\Delta \mathrm{Po}$ fracionamento $\left.=\mathrm{Po}_{\text {bic }}+\mathrm{Po}_{\text {hid }}+\mathrm{Po}_{\text {hid } 0,5}\right)$. Os resultados são mostrados no quadro 3, onde se observa que a depleção das formas inorgânicas ( $\Delta \mathrm{Pi}$ fracionamento) foi crescente conforme o histórico de adubação do solo, exceto para o tratamento $180 \mathrm{~kg} \mathrm{ha}^{-1}$ de $\mathrm{P}_{2} \mathrm{O}_{5}$, no qual ela foi discrepante dos outros resultados. Já para as formas orgânicas de $\mathrm{P}(\Delta \mathrm{Po}$ fracionamento), foi observado que a depleção foi semelhante em todos os tratamentos (aproximadamente $65 \mathrm{mg} \mathrm{kg}^{-1}$ ), mostrando que não houve mineralização preferencial de Po nos tratamentos com menor adição de adubos fosfatados e que ela se deveu, provavelmente, ao manejo utilizado no experimento (revolvimento, tamisagem e secagem a cada cultivo e, ainda, aplicação de calcário no oitavo cultivo). Entretanto, se expressos em termos percentuais (Quadro 3), observa-se que os cultivos provocaram a utilização das formas de $\mathrm{Pi}$ de modo crescente com o histórico de adubação, representando $24,9 \%$ no tratamento testemunha e $53,6 \%$ no trata-

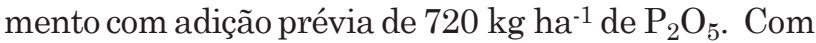
isso, o Po foi responsável por 75,1 \% da sustentação dos teores absorvidos no tratamento testemunha e por 46,4 \% no tratamento com a maior adição de $\mathrm{P}$.

A partir do ano de 2004, os Estados do Rio Grande do Sul e de Santa Catarina estabeleceram faixas de interpretação para os teores de P extraídos por resina trocadora de ânions em lâminas, sendo o nível crítico desse elemento fixado em $20 \mathrm{mg} \mathrm{dm}^{-3}$ (CQFSRS/SC, 2004). Por essa classificação, antes do início dos cultivos sucessivos dos solos utilizados no presente 
Quadro 3. Fósforo absorvido pelas plantas durante 15 cultivos sucessivos de amostras de um Latossolo Vermelho distroférrico típico em casa de vegetação e depleção dos teores de fósforo inorgânico, orgânico e total detectada pelo método de fracionamento de Hedley

\begin{tabular}{|c|c|c|c|c|c|c|}
\hline \multirow{2}{*}{$\begin{array}{c}\text { Tratamento }^{(1)} \\
\mathrm{P}_{2} \mathrm{O}_{5}, \mathrm{~kg} \mathrm{ha}^{-1}\end{array}$} & \multirow[t]{2}{*}{ P absorvido ${ }^{(2)}$} & \multirow{2}{*}{$\begin{array}{c}\Delta \mathbf{P} \text { fracionamento }{ }^{(3)} \\
\mathrm{mg} \mathrm{kg}^{-1}\end{array}$} & \multicolumn{2}{|c|}{$\Delta \mathrm{Pi}$ fracionamento ${ }^{(4)}$} & \multicolumn{2}{|c|}{$\Delta$ Po fracionamento ${ }^{(5)}$} \\
\hline & & & 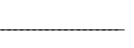 & $\%$ & $\mathrm{mg} \mathrm{kg}^{-1}$ & $\%$ \\
\hline 0 & 62,7 & 82,8 & 20,6 & 24,9 & 62,2 & 75,1 \\
\hline 180 & 70,2 & 85,2 & 6,2 & 7,8 & 79,0 & 92,2 \\
\hline 360 & 84,7 & 86,6 & 23,7 & 27,4 & 62,9 & 72,6 \\
\hline 540 & 90,0 & 125,1 & 61,7 & 49,3 & 63,4 & 50,7 \\
\hline 720 & 102,4 & 149,9 & 80,4 & 53,6 & 68,5 & 46,4 \\
\hline
\end{tabular}

${ }^{(1)}$ Doses de $\mathrm{P}$ adicionadas ao solo previamente ao experimento de cultivos sucessivos em casa de vegetação. ${ }^{(2)}$ Somatório das quantidades de $\mathrm{P}$ absorvido pelas plantas (parte aérea + raízes) nos 15 cultivos sucessivos. As espécies cultivadas foram milheto - Pennisetum americanum (cultivo 1); aveia-preta - Avena strigosa (cultivos 2, 3 e 4); milho - Zea mayz (cultivo 5); soja - Glycine max (cultivos 6, 7, 8, 9, 13, 14 e 15); crotalária - Crotalaria spectabilis (cultivo 10); centeio - Secale cereale (cultivos 11 e 12). ${ }^{(3)}$ Diferença entre os teores de $\mathrm{P}$ em todas as formas de $\mathrm{P}$ do fracionamento (somatório de todas as formas de $\mathrm{P}$ ) no solo original e naquele após 15 cultivos sucessivos. ${ }^{(4)}$ Diferença entre os teores das formas inorgânicas de P do fracionamento no solo original e naquele após 15 cultivos sucessivos. ${ }^{(5)}$ Diferença entre os teores das formas orgânicas de $\mathrm{P}$ do fracionamento no solo original e naquele após 15 cultivos sucessivos.

estudo, apenas aqueles com aplicação de 540 e $720 \mathrm{~kg} \mathrm{ha}{ }^{-1}$ de $\mathrm{P}_{2} \mathrm{O}_{5}$ encontravam-se acima do nível crítico (Quadro 1). Agrupando-se as amostras do solo por esse critério, naqueles inicialmente abaixo do nível crítico para as culturas $\left(0,180\right.$ e $360 \mathrm{~kg} \mathrm{ha}^{-1}$ de $\left.\mathrm{P}_{2} \mathrm{O}_{5}\right)$, as formas inorgânicas de $\mathrm{P}$ foram responsáveis por, em média, 20 \% do $\mathrm{P}$ absorvido, e as orgânicas, por 80 \% (Quadro 3). Já nas amostras inicialmente acima do nível crítico (540 e $720 \mathrm{~kg} \mathrm{ha}^{-1}$ de $\mathrm{P}_{2} \mathrm{O}_{5}$ ), as formas inorgânicas foram responsáveis por, em média, $51 \%$ do $\mathrm{P}$ absorvido, e as orgânicas, por 49 \%. Embora evidenciado no quadro 3 que a mineralização do Po foi independente do histórico de adubação fosfatada do solo, estando mais ligado a outros fatores de manejo experimental, cabe ressaltar que o Po representou a maior porção disponibilizada às plantas em sistema com baixas adições de fosfato, como observado por Gatiboni et al. (2005); na ausência de Po, as plantas provavelmente teriam seu crescimento impedido por deficiência de $\mathrm{P}$ antes do décimo quinto cultivo. Assim, embora liberando quantidades aquém das necessárias para o perfeito crescimento das culturas, as formas orgânicas de $\mathrm{P}$ constituem uma fonte imprescindível para manutenção de ecossistemas com baixos teores de Pi disponível (Adepetu \& Corey, 1976; Sharpley \& Smith, 1985; Guo \& Yost, 1998; Gatiboni et al., 2005).

\section{CONCLUSÕES}

1. Os teores de P extraídos por resina trocadora de ânions e, em seqüência, por bicarbonato de sódio $0,5 \mathrm{~mol} \mathrm{~L}^{-1}$ têm capacidade similar de tamponamento do $\mathrm{P}$ absorvido pelas plantas, podendo ser considerados formas lábeis de P.
2. Em longo prazo, todas as formas de $\mathrm{P}$ do solo atuam na biodisponibilidade do $\mathrm{P}$, porém a liberação de $\mathrm{P}$ pelas formas recalcitrantes acontece em quantidades e velocidade insuficientes para a absorção pelas plantas.

3. Em longo prazo, em solos com adição de fertilizantes fosfatados em quantidades suficientes para o crescimento e desenvolvimento das plantas, as formas inorgânicas e orgânicas de $\mathrm{P}$ têm capacidade semelhante de fornecer $\mathrm{P}$ para as plantas.

4. Em solos com baixa ou nenhuma adição de fertilizantes fosfatados, as formas orgânicas de P são as principais responsáveis pelo fornecimento deste nutriente às plantas.

\section{LITERATURA CITADA}

ADEPETU, J.A. \& COREY, R.B. Organic phosphorus as a predictor of plant-available phosphorus in soils of Southern Nigeria. Soil Sci., 122:159-164, 1976.

BECK, M.A. \& SANCHES, P.A. Soil phosphorus fraction dynamics during 18 years of cultivation on a Typic Paleudult. Soil Sci., 34:1424-1431, 1994.

BROOKES, P.C. \& POWLSON, D.S. Preventing phosphorus losses during perchloric acid digestion of sodium bicarbonate soil extracts. J. Sci. Food Agric., 32:671-674, 1981.

CHEN, C.R.; CONDRON, L.M.; DAVIS, M.R. \& SHERLOCK, R.R. Phosphorus dynamics in the rhizosphere of perennial ryegrass (Lolium perenne L.) and radiata pine (Pinus radiata D. Don.). Soil Biol. Biochem., 34:487-499, 2002. 
COMISSÃO DE FERTILIDADE DO SOLO - CFSRS/SC Recomendação de adubação e calagem para os Estados do Rio Grande do Sul e Santa Catarina. 3.ed. Passo Fundo, SBCS/NRS/Embrapa/CNPT, 1994. 224p.

COMISSÃO DE QUÍMICA E FERTILIDADE DO SOLO CFSRS/SC. Manual de recomendação de adubação e de calagem para os Estados do Rio Grande do Sul e Santa Catarina. 10.ed. Porto Alegre, SBCS/NRS, 2004. 400p.

CONDRON, L.M.; GOH, K.M. \& NEWMAN, R.H. Nature and distribution of soil phosphorus as revealed by a sequential extraction method followed by ${ }^{31} \mathrm{P}$ nuclear magnetic resonance analysis. J. Soil Sci., 36:99-207, 1985.

CONTE, E. Atividade de fosfatase ácida e formas de acumulação de fosfato em solo no sistema plantio direto. Porto Alegre, Universidade Federal do Rio Grande do Sul, 2001. 65p. (Tese de Mestrado)

CROSS, A.F. \& SCHLESINGER, W.H. A literature review and evaluation of the Hedley fractionation: Applications to the biogeochemical cycle of soil phosphorus in natural ecosystems. Geoderma, 64:197-214, 1995.

DALAL, R.C. Soil organic phosphorus. Adv. Agron., 29:83 $117,1977$.

DAROUB, S.H.; PIERCE, F.J. \& ELLIS, B.G. Phosphorus fractions and fate of phosphorus-33 in soils under plowing and no-tillage. Soil Sci. Soc. Am. J., 64:170-176, 2000.

DICK, W.A. \& TABATABAI, M.A. Determination of orthophosphate in aqueous solutions containing labile organic and inorganic phosphorus compounds. J. Environ. Qual., 6:82-85, 1977.

GATIBONI, L.C.; RHEINHEIMER, D.S.; FLORES, A.F.C.; ANGHINONI, I.; KAMINSKI, J. \& LIMA, M.A.S. Phosphorus forms and availability assessed by ${ }^{31} \mathrm{P}-\mathrm{NMR}$ in successive cropped soil. Comm. Soil Sci. Plant Anal., $36: 2625-2640,2005$.

GUO, F. \& YOST, R.S. Partitioning soil phosphorus into three discrete pools of differing availability. Soil Sci., 163:822833, 1998.

HEDLEY, M.J.; STEWART, J.W.B. \& CHAUHAN, B.S. Changes in inorganic and organic soil phosphorus fractions induced by cultivation practices and by laboratory incubations. Soil Sci. Soc. Am. J., 46:970-976, 1982.

HENRIQUEZ, C. \& KILLORN, R. Soil P forms and P uptake under intensive plant growth in the greenhouse. Agron. Costarricence, 29:83-97, 2005.
MAROKO, J.B.; BURESH, R.J. \& SMITHSON, P.C. Soil phosphorus fractions in unfertilized fallow-maize systems on two tropical soils. Soil Sci. Soc. Am. J., 63:320-326, 1999.

MURPHY, J. \& RILEY, J.P. A modified single solution method for the determination of phosphate in natural waters. Anal. Chim. Acta, 27:31-36, 1962.

OLSEN, S.R. \& SOMMERS, L.E. Phosphorus. In: PAGE, A.L.; MILLER, R.H. \& KEENEY, Q.R., eds. Methods of soil analysis. Chemical and microbiological properties. 2.ed. Madison, SSSA. 1982. Part 2. p.403-430.

PARFITT, R.L. Anion adsorption by soils and soil materials. Adv. Agron., 30:01-46, 1978.

RHEINHEIMER, D.S. Dinâmica do fósforo em sistemas de manejo de solos. Porto Alegre, Universidade Federal do Rio Grande do Sul, 2000. 210p. (Tese de Doutorado)

RHEINHEIMER, D.S.; CONTE, E. \& ANGHINONI, I. Formas de acumulação de fósforo pela aplicação de fosfato em solo no sistema plantio direto. R. Bras. Ci. Solo, 27:893900, 2003.

RHEINHEIMER, D.S. \& ANGHINONI, I. Distribuição do fósforo inorgânico em sistemas de manejo de solo. Pesq. Agropec. Bras., 36:151-160, 2001.

SHARPLEY, A.N. \& SMITH, S.J. Fractionation of inorganic and organic phosphorus in virgin and cultivated soils. Soil Sci. Soc. Am. J., 49:127-130, 1985.

SHARPLEY, A.N.; TIESSEN, H. \& COLE, C.V. Soil phosphorus forms extracted by soil tests as a function of pedogenesis. Soil Sci. Soc. Am. J., 51:362-365, 1987.

STEWART, J.W.B. \& TIESSEN, H. Dynamics of soil organic phosphorus. Biogeochemistry, 4:41-60, 1987.

TEDESCO, M.J.; GIANELLO, C.; BISSANI, C.A.; BOHNEN, H. \& VOLKWEISS, S.J. Análise de solo, plantas e outros materiais. Porto Alegre, Universidade Federal do Rio Grande do Sul, 1995. 174p.

TIESSEN, H.; STEWART, J.W.B. \& COLE, C.V. Pathways of phosphorus transformations in soils of differing pedogenesis. Soil Sci. Soc. Am. J., 48:853-858, 1984.

UNITED STATES ENVIRONMENTAL PROTECTION AGENCY - USEPA. Methods of chemical analysis for water and wastes. Cincinnati, 1971. 312p.

UNITED STATES ENVIRONMENTAL PROTECTION AGENCY - USEPA. Methods of chemical analysis for water and wastes. Cincinnati, 1971. 312p. 